OPEN ACCESS

Edited by:

Thomas Frazier,

John Carroll University, United States

Reviewed by:

Arun Karpur,

Autism Speaks, United States

*Correspondence:

Yun-Ju Chen

cheny793@mcmaster.ca

Specialty section

This article was submitted to

Autism,

a section of the journal

Frontiers in Psychiatry

Received: 02 December 2021

Accepted: 31 January 2022

Published: 25 February 2022

Citation:

Chen Y-J, Duku E and Georgiades S

(2022) Rethinking Autism Intervention

Science: A Dynamic Perspective.

Front. Psychiatry 13:827406.

doi: 10.3389/fpsyt.2022.827406

\section{Rethinking Autism Intervention Science: A Dynamic Perspective}

\author{
Yun-Ju Chen*, Eric Duku and Stelios Georgiades \\ Department of Psychiatry and Behavioural Neurosciences, McMaster University, Hamilton, ON, Canada
}

Recent advances in longitudinal methodologies for observational studies have contributed to a better understanding of Autism as a neurodevelopmental condition characterized by within-person and between-person variability over time across behavioral domains. However, this finer-grained approach to the study of developmental variability has yet to be applied to Autism intervention science. The widely adopted experimental designs in the field-randomized control trials and quasi-experimental designs - hold value for inferring treatment effects; at the same time, they are limited in elucidating what works for whom, why, and when, given the idiosyncrasies of neurodevelopmental disorders where predictors and outcomes are often dynamic in nature. This perspective paper aims to serve as a primer for Autism intervention scientists to rethink the way we approach predictors of treatment response and treatment-related change using a dynamic lens. We discuss several empirical gaps, and potential methodological challenges and opportunities pertaining to: (1) capturing finer-grained treatment effects in specific behavioral domains as indexed by micro-level within-person changes during and beyond intervention; and (2) examining and modeling dynamic prediction of treatment response. Addressing these issues can contribute to enhanced study designs and methodologies that generate evidence to inform the development of more personalized interventions and stepped care approaches for individuals on the heterogeneous spectrum of Autism with changing needs across development.

Keywords: autism (ASD), intervention outcome, developmental trajectories, time-varying (TV), longitudinal, prediction

\section{INTRODUCTION}

Over the past decade, the field of Autism intervention science has made significant advances that led to promising evidence on improving the developmental outcomes of individuals with Autism (1). However, methodological concerns such as small sample size, detection bias related to limited informant types and objective outcome measures, and restricted trial contexts, continue to limit the replicability and generalizability of these findings (1-3). Recent meta-analytic studies revealed empirical gaps in the prediction of differential treatment response and mechanisms through which treatments work, potentially due to limited statistical power and discrepancies in designs and reporting practices across studies $(1,4)$.

Certain conceptual limitations in manipulating and evaluating treatment-related change could also be a barrier to advancing personalized care in Autism. Specifically, Autism intervention science has historically relied on traditional randomized control trials (RCTs) and quasi-experimental methodologies that often do not account for the heterogeneous and dynamic nature of Autism. 
While being useful in yielding causal inferences of treatment effects, RCTs evaluating Autism interventions use the process of randomization to "control" statistically for the possible influence of static "confounding" factors at baseline not under direct experimental control. Also, treatment response in RCTs is often determined by comparing group (experimental vs. control) differences or subtracting placebo response from the overall response, thus being limited in assessing individual-level treatment response (5). This is particularly relevant to Autism, as we know from observational longitudinal studies that variable developmental trajectories can be identified among autistic individuals over the life span in several behavioral domains that are often the targets of Autism intervention studies, such as core symptoms of Autism, adaptive functioning, IQ, and challenging behavior (6-9). The waxing and waning of target outcomes across development may contribute to the variable treatment response, but it is difficult to differentiate the sources of variability under the traditional experimental designs. For instance, some target treatment outcomes may decrease over a longer time span as individuals grow out of certain behaviors (e.g., from non-verbal to verbal communication), resulting in an artifact of reduced treatment response (10). Although the traditional RCT design has proven to be invaluable and thought of as the gold standard of evidence for the study of other-mostly physicaldisorders, the derived findings are often limited in generalization beyond the trial sample given the restrictions mentioned above (11). Considering the idiosyncrasies of neurodevelopmental disorders where behavioral manifestations are often dynamic and heterogeneous in nature, there is a need to rethink how we approach the study of Autism intervention ingredients, including both predictors and outcomes, to better elucidate what works for whom, why, and when.

\section{CAPTURING INDIVIDUAL-LEVEL VARIABILITY IN TREATMENT OUTCOMES}

In classical RCTs and quasi-experimental designs, it is common to collect outcome data at pre- and post-treatment, sometimes with post-treatment follow-up. While this satisfies the purpose of inferring whether the treatment is more effective than placebo, the "black box" of what happens during treatment remains unopened (see Figure 1). Further, the treatment-related change is often treated as a "chunk" averaged across individuals (e.g., average treatment effect, average effects on the treated) rather than a continuous process over time (e.g., withinperson change) for each individual. While strategies such as subgroup analysis and propensity scores based on a priori groupings (e.g., sex) can be used for addressing heterogeneous treatment effects (12), it could still be problematic when observations do not correspond to individual experience or behavior in a non-ergodic (i.e., non-stationary and variable) behavioral change process in the real world, thus limiting the generalization and replication of the findings $(13,14)$. It also poses challenges in differentiating between individual treatment response and random variability that may bias the evaluation of treatment response. Some potential sources of bias include natural fluctuations of treatment outcomes, response bias (e.g., tendency to report favorable outcomes), practice effects, statistical artifacts (e.g., regression to the mean, ceiling/floor effect) (15). Finally, although randomization helps to increase the internal validity of group comparisons by making the factors associated with unobserved uncertainty equitably distributed across the treatment and the control groups, meaningful individual variability might also be distributed across the two groups. When sample size is small and/or individual variability is not well addressed with appropriate analytical approaches (e.g., accounting for within-group variations), there could be a higher probability of type II errors and thus reduced power to detect effects (16).

Although recent advances in longitudinal designs and analyses for observational studies have contributed to a better understanding of the heterogeneity of progression of Autismrelated phenotypes both within and between individuals and over time-i.e., chronogeneity $(17,18)$ - how treatment outcomes are approached in Autism intervention research remains limited in addressing individual-level variability with respect to time. To date, there are only a handful of larger-scale intervention studies that describe developmental trajectories of intervention outcomes using approaches accounting for both within-person and between-person differences, such as multilevel modeling and latent growth curve analysis. In an RCT study (10), variable trajectories of joint attention behaviors were observed among a group of preschool-aged children diagnosed with Autism over the course of social communication intervention and 5year follow-up, where the change patterns were associated with treatment assignment and diagnostic status at the exit. A recent observational study (19) reported an overall increase across diverse language trajectories between the entry and exit of an early intensive behavioral intervention (EIBI) program among preschool-aged children with Autism, with steeper improvements predicted by younger age, higher cognitive abilities, and lower symptom severity at baseline. Another observational study (20) examined the growth curve of autistic children's developmental outcomes across several time-points during applied behavior analysis (ABA) intervention and found that symptom severity, primary language spoken at home, and child's sex, but not treatment intensity and age of entry, were significant predictors of growth rates in certain outcomes during the intervention. Along with another observational EIBI study for children with Autism (21), different rates of improvement in treatment outcomes were observed across timepoints during the period of intervention. For instance, many children tended to show patterns of exponential negative growth (faster improvement in the beginning followed by decelerated progress after). These findings suggest that treatment-related change is developmentally variable, and differences in baseline characteristics within treatment groups can be associated with various treatment responses or lead to different treatment outcomes. Also, the rate of change may vary during and beyond the intervention, potentially in a non-linear trend that is often hard to observe with limited data points. This indicates that there may be a time window for certain groups of people to better respond to the treatment. 


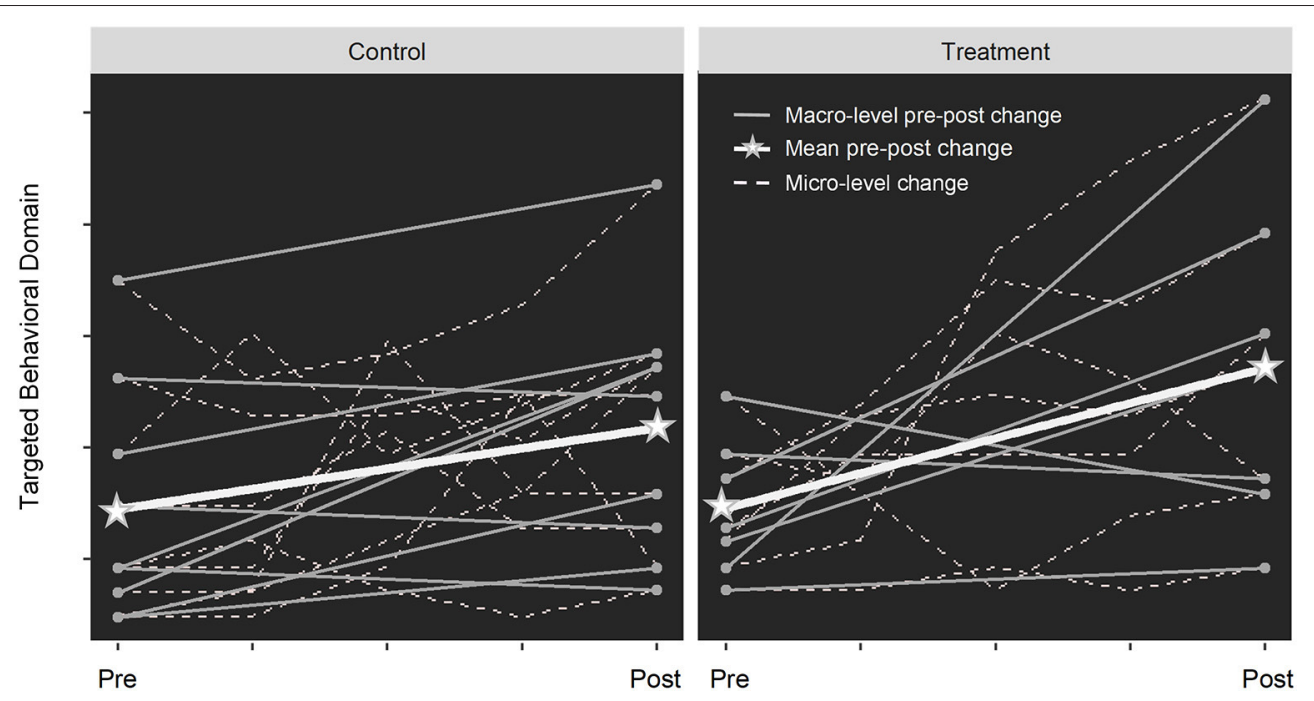

FIGURE 1 | The "black box" of treatment effect in RCTs. In an RCT design, participants are randomized to treatment or control groups with matched baseline characteristics. When only calculating the average change from pre- to post-treatment (thick white lines), the larger increase observed in the treatment group may lead to the conclusion that the treatment is effective (assuming significant group difference), despite the individual-level heterogeneous response (gray lines). When breaking the treatment period into smaller intervals, the micro-level change (dashed lines) reveals that the rate of change varies across individuals over time, indicating time-varying treatment effects. Regarding "opening the black box", we are not referring to unblinding the clinical trial procedures, but rather adopting study designs (e.g., more frequent data collection with more refined outcome measures over a longer time span) and analytical approaches (e.g., trajectory analyses) that allow for examining the finer-grained changes in treatment response.

Despite emerging evidence on the complex patterns of treatment-related change, more research that captures finegrained variability over time is needed for informing personalized intervention in Autism. The fundamental issue might lie in the imbalance between simplicity and complexity when approaching treatment-related change with a lack of respect to the role of time in the risk and resilience process (22), thus limiting the field from yielding robust, meaningful, and translatable findings. Below we discuss some empirical gaps, methodological challenges, and opportunities that could be drawn from other fields, as well as an illustrative example for autism researchers to plan for "next steps".

\section{Research Questions/Hypotheses}

While Autism intervention science often poses the question of what works for whom, and why/when the change happened, the main research question tested empirically is whether the change happened due to the specific treatment. Although the latter question is foundational for demonstrating the effectiveness of treatment, it may not be sufficient in providing generalizable information on applications outside the clinical-trial settings where more variability related to individual differences or time is expected. Aside from confirming whether the treatment works, we may also explore the overall shape (i.e., progression) of change in specific proximal or distal treatment outcomes, when the greatest amount of change or inconsistent rate of change occurs, and how these patterns of change are associated with certain individual characteristics. Answers to these questions would inform the development of more tailored treatment programs that are more targeted and better timed for optimal response.

\section{Design/Measurement}

These types of research questions highlight the need for refining the tracking of treatment outcomes through appropriate study designs, including:

- More frequent measurement occasions at shorter timescales to capture finer-grained behavioral change processes of treatment targets: While intensive data collection of proximal treatment targets is a common practice in EIBI, individual variability in behavioral change has rarely been addressed. Recently, intensive longitudinal (IL) methods, such as ecological momentary assessment, experience sampling, and daily diary, have been increasingly adopted in the field of psychopathology to better capture the temporal dynamics of symptoms and functions, thus allowing for better elucidation of treatment effects mechanisms $(23,24)$. While it remains challenging to collect longitudinal data with validated tools in behavioral research, the recent advance in remote monitoring and telehealth methods as well as the use of accelerated longitudinal design could facilitate the feasibility of more intensive behavioral data collection during clinical trials $(24,25)$.

- More refined and psychometrically validated behavioral constructs as treatment outcome measures: Given the multidimensional clinical features of Autism and associated challenges, it would be useful to have measures that capture specific domains (or sub-domains) of targeted outcomes and other key neurobehavioural constructs (e.g., specific joint attention skills instead of a general social communication composite score) at multiple time points to be able to examine the interplay of different treatment outcome domains 
over time, as well as to better account for the heterotypic development (i.e., age-dependent behavioral manifestations) of outcomes during long-term follow-up.

\section{Analysis}

Future Autism intervention research may benefit from applying the learnings achieved in observational studies describing heterogeneous developmental trajectories. Specifically, analytical approaches for studying between-person differences in withinperson change (e.g., latent growth modeling and multilevel modeling), and person-centered approaches for identifying homogeneous subgroups (e.g., growth mixture modeling), can contribute to better capturing individual treatment-related change over time. These approaches allow for addressing a variety of development-related complexities, such as non-linear trajectories, time-varying predictors, and interactions across multiple treatment outcome domains. They are also flexible in dealing with some common challenges in intervention studies, such as missing data and non-normally distributed measures (26). It should be noted that these approaches often require at least three time-points of panel data to estimate linear latent trajectories or linear random effects and four time-points for capturing non-linear within-person and between-person changes (27).

\section{Illustrative Example}

te Brinke et al. (28) recruited a total of 108 adolescents with elevated externalizing behavior, who were randomized to either a treatment (emotion regulation training) group or a control group. Emotion regulation strategies and externalizing problems were assessed at baseline and at two treatment phases. At each phase (spanning 3-7 weeks), self-reports of emotion regulation difficulties and aggression were collected weekly via smartphones. Aside from examining the group differences in distal treatment outcomes (i.e., emotion regulation strategies and externalizing problems), this design allowed to examine the effect of treatment manipulation (alternating the sequence of cognitive or behavioral approaches) on proximal outcomes (i.e., emotion regulation difficulties and aggression) by modeling their piecewise trajectories across individuals in the treatment group. Similarly, autism researchers may apply such design to examine the trajectories of distal treatment outcomes (e.g., expressive language) between groups as well as finer-grained with-person changes in proximal treatment outcomes (e.g., specific joint attention skills) through more intensive data collection. Such an approach would also allow for examining the potential effect of changing intervention ingredients (e.g., sequence and dosage), which would be particularly useful under an adaptive intervention design.

\section{MODELING DYNAMIC PREDICTION OF TREATMENT RESPONSE}

As we continue to advance our work on describing treatmentrelated change over time, it is also important to identify predictors of "more responsive" trajectories (e.g., higher rates of improvement, longer maintenance of treatment effects). As reported in the intervention studies mentioned above $(19,20)$, some child demographics (e.g., age, sex) and characteristics at baseline (e.g., IQ, level of symptoms) were associated with different trajectories during and/or after the intervention. What remains unclear, however, is the dynamic processes between predictors and treatment outcomes underlying these variable trajectories. Conventionally, predictors and their effects are treated as "static" based on the assumptions that, for example, cognitive and language skills do not change beyond the baseline and their effects on intervention outcomes hold constant over time. However, as demonstrated by many longitudinal studies, the outcomes and predictors of interest are often not static [e.g., IQ and symptoms of Autism; (8)] and may have dynamic associations with each other over the period of observation [e.g., language and social skills; (29)] among individuals with Autism. Moreover, major life changes, such as a transition to school and the COVID-19 pandemic, may "disrupt" children's trajectory outcomes and their associations with predictors $(30,31)$. Changing intervention components (e.g., types, dose, duration, intensity of treatment) may also influence such dynamics. Recently, adaptive intervention approaches (e.g., sequential multiple-assignment randomized trials, SMARTs) have represented a promising strategy to personalize Autism intervention (32), where participants are randomized into different sequences of intervention options according to their response to treatment. These "smarter" intervention approaches require "smarter" analytic methods to better address treatmentrelated change. And even in the case of predictors that are invariant in nature (e.g., sex), the magnitude of their predictive effect may still vary across the course of intervention and/or development [e.g., interactions between sex and age for comorbid symptoms in children with Autism; (33)]. All these complexities regarding the prediction of treatment-related change point to the need for more "time-sensitive" approaches, such as dynamic prediction modeling that allows for examining time-varying effects on treatment outcomes (see Figure 2).

The concept of dynamic prediction is not new to the field of psychopathology, which has been adopted in intervention studies for alcohol or drug addiction and affective disorders [e.g., $(34,35)]$ as well as in non-intervention studies such as the prediction of mental disorder onset and progression (36, 37). The idea behind the dynamic prediction is to approach psychopathology as a system rather than as a category (37) through capturing the reciprocal relation between trajectories of interest (e.g., treatment outcomes) and their etiologically and clinically relevant time-varying predictors (34). Recently, as a response to the impact of COVID-19, a dynamic clinical prediction model has been proposed to adapt to the constantly evolving healthcare system, where predictors as associated with changes in population demographics, prevalence of disease, and clinical practice paradigms are taken into account for decisionmaking $(38,39)$. From an analytic perspective, changes that arise over time (beyond experimental control) may introduce uncertainty to prediction models and result in "calibration drift” (i.e., less accurate predictive ability over time) (40). Thus, establishing prediction models with only baseline predictors may under-utilize the available information and thus limit predictive 


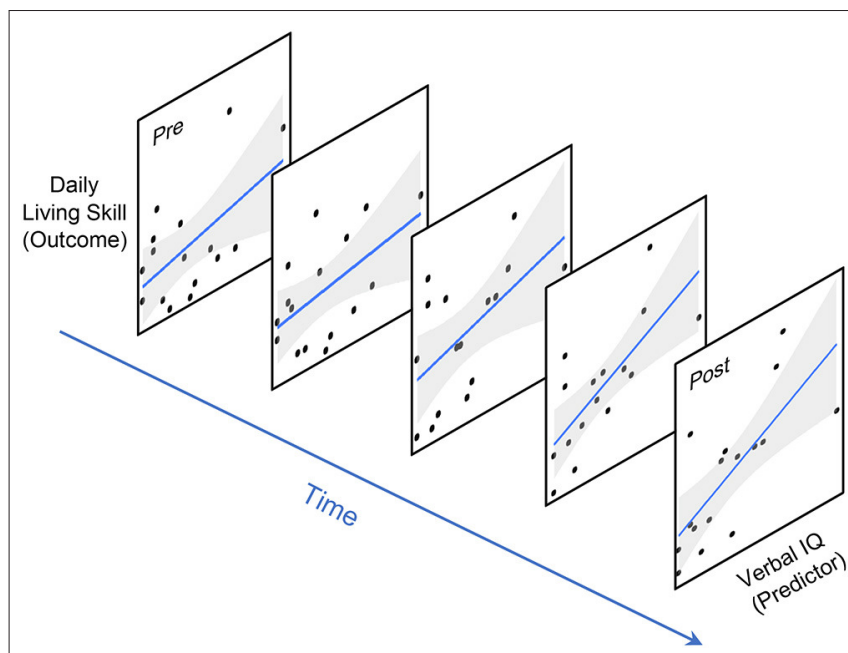

FIGURE 2 | Time-varying prediction of treatment outcome. Conventional linear regression approaches assume the effect of predictors/covariates (e.g., IQ) on treatment outcomes (e.g., adaptive skills) to be static or constant over time. In contrast, a time-varying prediction model estimates their association as a function of time.

ability and replicability (41). While our current knowledge about predictors of treatment outcomes in Autism remains inconclusive due to several conceptual and methodological limitations, such as a lack of theory-driven models with attention to individual differences (42), the missing piece of "time" may be a major factor underpowering the detection of meaningful effects.

The concept of dynamic prediction also applies to the study of treatment mediation given the nature of mediators as outcome predictors. Mediation is commonly studied with regressionbased approaches in intervention studies to understand treatment processes and mechanisms. Such approaches often assume mediation effects to be linear and thus ignore that independent variable, outcome variable and mediator are typically not in a strictly unidirectional and static relation, but instead in a bidirectional relation that may change over time (42). As demonstrated in a large-scale RCT study with longterm follow-up for a parent-mediated social communication intervention targeting children with Autism (43), while the treatment effect on parental synchrony (mediator) attenuated over time, the treatment effect on child outcomes did sustain at follow-up, indicating that the mediation mechanism could vary across different stages of intervention. Such finding also supports the theoretical foundation behind development-based intervention approaches (e.g., naturalistic developmental behavioral interventions), in which developmentally-appropriate precursor skills are targeted to improve developmental outcomes. Thus, a move from static to dynamic approaches of examining predictive effects, including moderation and mediation, would not only facilitate our understanding of why and when treatment response becomes differential across individuals, but also better reflect the rationale of developmentally grounded intervention approaches.

Given the advances across the broader field of psychopathology in addressing the dynamic nature of the predictor-outcome relation, as contrasted with the common practice of studying this interplay as static in Autism intervention science, we suggest that future research may want to identify time-varying predictors or covariates of treatment outcomes based on theory and existing evidence. Here we raise some potential challenges and opportunities regarding dynamic prediction of treatment outcomes, along with an illustrative example that might be applied to Autism intervention research.

\section{Research Questions/Hypotheses}

As discussed above, some common baseline predictors, such as cognitive and language skills, may change over time and have a dynamic relation with each other and with treatment outcomes. Mediating effects could also vary across time, such as the effect of parent responsiveness on child's treatment outcomes during parent-mediated intervention vs. follow-up. In this regard, some examples of "time-sensitive" research questions that can be asked include: (1) When (e.g., 1 month upon entry, 6 months after exit) does the treatment effect become active or reduced? (2) How do individuals with certain characteristics or in different contexts (e.g., verbal vs. non-verbal, various intervention elements, levels of environmental support) differentially respond to the treatment over time (i.e., time-varying moderation of treatment outcome)? (3) Does the mediating effect on treatment outcome (e.g., parental responsiveness on child's social response) vary over time? (4) When do two or multiple behavioral domains of interest (e.g., core symptoms and comorbidities) become "decoupled" as the result of the intervention? These "time-sensitive" research questions could yield findings that fill the empirical gaps in the Autism intervention research regarding treatment timing and underlying mechanisms.

\section{Design/Measurement}

Modeling dynamic prediction of treatment response requires repeated data collections of (lagged or concurrent) outcome and predictor variables with adequate coverage across the time span. And as with any longitudinal analysis, the assumption of measurement invariance across time should be met. We note that some challenges which have hampered Autism intervention research historically, such as the burden of repeated measurements on both participants and assessors, low recruitment numbers and high attrition rates (that lead to smaller sample sizes), are still relevant here. However, lessons and opportunities could be drawn from cross-site collaborations and consortiums for genomic and biomarker data that have been developed over the past decades in the field of Autism research $(44,45)$ for increasing sample sizes as well as enhancing data sharing and harmonization of clinical trial data, which would allow researchers to address more complex but relevant research questions. The Autism research community needs to work together to make it possible to identify meaningful predictors of treatment response through precision approaches (3).

\section{Analysis}

As a direct extension from the widely adopted cross-lagged panel models in longitudinal studies, the incorporation of time-varying covariates could be achieved by specifying random intercept factors that represent the person-specific deviations from mean 
trajectories at a specific time-point (46). A similar idea can be also applied to multivariate latent curve modeling with structured residuals that capture time-specific within-person differences in the association of multiple trajectories (e.g., the association between trajectories of treatment outcome and predictor) (47). Other novel methods which have been increasingly used in the field of psychopathology include time-varying effect modeling [TVEM; (48)], dynamic structural equation modeling [DSEM; (49)], and joint modeling (50). A shared characteristic of these methods is the non-parametric or semi-parametric estimation of regression coefficients for time-series data without a priori constraints on underlying trajectories and shapes of coefficient functions. These methods have been applied to intervention and prevention for addiction and affective disorders [e.g., (51-53)], as well as detection of transition to psychosis [e.g., (54)], and thus may be useful candidates to be applied and tested in Autism intervention science. Survival analytical approaches that are widely adopted in medical and epidemiological research, such as Cox proportional-hazards regression models, which assume loglinearity in covariates, could also be used to examine the timevarying effects of covariates (55). Finally, Bayesian approaches could be applied to handle time-varying coefficient models with greater complexity (e.g., multiple random effects) (56).

\section{Illustrative Example}

Wright et al. (51) examined the impact of co-occurring anxiety on depression treatment outcomes among 78 outpatients over the course of psychotherapy. The patients received either traditional psychotherapy for depression or a variant that also targets anxiety. Depression and anxiety symptoms were assessed by clinicians at each of the 16 weekly treatment sessions. Instead of using baseline anxiety as a predictor, the researchers examined the dynamic associations (i.e., time-specific coefficients) between anxiety and depression using TVEM to clarify whether anxiety and depression "decoupled" as treatment proceeds. They also examined whether the time-varying associations differ between groups who received different versions of treatment at certain time-points. Autism researchers could also apply a similar approach to explore research questions related to co-occurring symptoms (e.g., repetitive and restricted behaviors and anxiety) during behavioral interventions, parent-child dyads (e.g., parent responsiveness and child's joint attention skills) during parentmediated interventions, or inclusion of predictors that may change in nature (e.g., cognitive and language skills). This would allow for elucidating how and when the covariates of interest contribute to treatment targets or interact with intervention elements at an individual level.

\section{CONCLUSION}

We discussed here two important empirical gaps in Autism intervention science that, to date, has been relying on observational or experimental designs (including RCTs) predominantly characterized by: (1) evaluating treatment effects based on group comparisons of mean pre-post changes in general outcome domains; and (2) studying prediction of treatment outcomes as a static phenomenon. The failure to measure more real-time treatment response, particularly under a pre-post design, may lead to a biased inference of treatment effects. Moreover, while it is reasonable to "control for" static predictors of intervention outcomes, the findings should be cautiously interpreted given the untested assumption that the predictors pose effects on the target outcomes that do not vary in strength over time. This, however, may undermine the predictive accuracy of treatment response and limit the generalization of findings to autistic populations with heterogeneous developmental profiles in real-world contexts. Given the dynamic and developmental nature of psychopathology (57), such as the gene-environment interplay that may impact developmental outcomes in Autism $(58,59)$, it is important to take temporal and contextual dimensions of treatment effects into account to elucidate why and when the intervention works or does not work. We discussed several methodological challenges and potential solutions for addressing these empirical gaps when designing Autism intervention studies. Adopting a dynamic lens can help researchers and clinicians to better understand the adaptive developmental processes to positive or negative changes associated with intervention or environment (59), whose importance is underscored by the pandemic's significant impacts on autistic individuals and their families $(31,60)$. As the field is entering the era of stepped and personalized healthcare (61), there is a need to pause, rethink, and discuss an intervention research agenda that better addresses the developmental and dynamic nature of Autism, and to adopt methodological approaches that support the shift of focus from macro to micro-level change, as well as from static to dynamic prediction of change. Such a paradigm shift would contribute to the refinement of personalized interventions tailored to heterogeneity across development (i.e., chronogeneity) so that interventions and services could be delivered to autistic individuals and their families in a timely, targeted, and adaptive manner.

\section{DATA AVAILABILITY STATEMENT}

The original contributions presented in the study are included in the article/supplementary material, further inquiries can be directed to the corresponding author/s.

\section{AUTHOR CONTRIBUTIONS}

SG and Y-JC contributed to the conception of this manuscript. Y-JC drafted the manuscript with comments and edits from ED and SG. The final version of the manuscript was read and approved by all the authors.

\section{FUNDING}

This work was supported by the McMaster Children's Hospital Chair in Autism and Neurodevelopment (to SG) and the Offord Center for Child Studies postdoctoral fellowship (to Y-JC). 


\section{REFERENCES}

1. Sandbank M, Bottema-Beutel K, Crowley S, Cassidy M, Dunham K, Feldman JI, et al. Project AIM: autism intervention meta-analysis for studies of young children. Psychol Bull. (2020) 146:1-29. doi: 10.1037/bul0000215

2. Green J, Garg S. Annual research review: the state of autism intervention science: progress, target psychological and biological mechanisms and future prospects. J Child Psychol Psychiatry. (2018) 59:424-43. doi: 10.1111/jcpp. 12892

3. Simonoff E. Commentary: randomized controlled trials in autism spectrum disorder: state of the field and challenges for the future. J Child Psychol Psychiatry. (2018) 59:457-9. doi: 10.1111/jcpp.12905

4. Crank JE, Sandbank M, Dunham K, Crowley S, Bottema-Beutel K, Feldman $\mathrm{J}$, et al. Understanding the effects of naturalistic developmental behavioral interventions: a project aim meta-analysis. Autism Res. (2021) 14:81734. doi: 10.1002/aur.2471

5. Senn S. Individual response to treatment: is it a valid assumption? BMJ. (2004) 329:966-8. doi: 10.1136/bmj.329.7472.966

6. Smith LE, Maenner MJ, Seltzer MM. Developmental trajectories in adolescents and adults with autism: the case of daily living skills. J Am Acad Child Adolesc Psychiatr. (2012) 51:622-31. doi: 10.1016/j.jaac.2012.03.001

7. Szatmari P, Georgiades S, Duku E, Bennett TA, Bryson S, Fombonne E, et al. Developmental trajectories of symptom severity and adaptive functioning in an inception cohort of preschool children with autism spectrum disorder. JAMA Psychiatry. (2015) 72:276. doi: 10.1001/jamapsychiatry.2014.2463

8. Simonoff E, Kent R, Stringer D, Lord C, Briskman J, Lukito S, et al. Trajectories in symptoms of autism and cognitive ability in autism from childhood to adult life: findings from a longitudinal epidemiological cohort. J Am Acad Child Adolesc Psychiatr. (2020) 59:1342-52. doi: 10.1016/j.jaac.2019.11.020

9. Stringer D, Kent R, Briskman J, Lukito S, Charman T, Baird G, et al. Trajectories of emotional and behavioral problems from childhood to early adult life. Autism. (2020) 24:1011-24. doi: 10.1177/1362361320908972

10. Gulsrud AC, Hellemann GS, Freeman SFN, Kasari C. Two to ten years: Developmental trajectories of joint attention in children with ASD who received targeted social communication interventions. Autism Res. (2014) 7:207-15. doi: 10.1002/aur.1360

11. Deaton A, Cartwright $\mathrm{N}$. Understanding and misunderstanding randomized controlled trials. Soc Sci Med. (2018) 210:221. doi: 10.1016/j.socscimed.2017.12.005

12. Kravitz R, Duan N, Braslow J. Evidence-based medicine, heterogeneity of treatment effects, and the trouble with averages. Milbank Q. (2004) 82:66187. doi: 10.1111/j.0887-378X.2004.00327.x

13. Fisher AJ, Medaglia JD, Jeronimus BF. Lack of group-to-individual generalizability is a threat to human subjects research. Proc Natl Acad Sci USA. (2018) 115: doi: 10.1073/pnas.1711978115

14. Heino MT, Knittle K, Noone C, Hasselman F, Hankonen N. Studying behaviour change mechanisms under complexity. Behav Sci. (2021) 11:77. doi: 10.3390/bs11050077

15. Kelley JM, Kaptchuk TJ. Group analysis versus individual response: the inferential limits of randomized controlled trials. Contemp Clin Trials. (2010) 31:423-8. doi: 10.1016/j.cct.2010.07.003

16. Chin R, Lee BY. Principles and Practice of Clinical Trial Medicine. London: Elsevier (2008).

17. Georgiades S, Bishop SL. Frazier T. Editorial perspective: Longitudinal research in autism - introducing the concept of 'chronogeneity'. J Child Psychol Psychiatry. (2017) 58:634-6. doi: 10.1111/jcpp. 12690

18. Lombardo MV, Lai M-C, Baron-Cohen S. Big data approaches to decomposing heterogeneity across the autism spectrum. Mol Psychiatry. (2019) 24:1435-50. doi: 10.1038/s41380-018-0321-0

19. Frazier TW, Klingemier EW, Anderson CJ, Gengoux GW, Youngstrom EA, Hardan AY, et al. longitudinal study of language trajectories and treatment outcomes of early intensive behavioral intervention for autism. J Autism Dev Disord. (2021) 51:4534-50. doi: 10.1007/s10803-021-04900-5

20. Tiura M, Kim J, Detmers D, Baldi H. Predictors of longitudinal ABA treatment outcomes for children with autism: a growth curve analysis. Res Dev Disabil. (2017) 70:185-97. doi: 10.1016/j.ridd.2017.09.008

21. Virues-Ortega J, Rodríguez V, Yu CT. Prediction of treatment outcomes and longitudinal analysis in children with autism undergoing intensive behavioral intervention. Int J Clin Health Psychol. (2013) 13:91-100. doi: 10.1016/S1697-2600(13)70012-7

22. Lerner RM, Schwartz SJ, Phelps E. Problematics of time and timing in the longitudinal study of human development: theoretical and methodological issues. Hum Dev. (2009) 52:44-68. doi: 10.1159/000189215

23. Hamaker EL, Wichers M. No time like the present. Curr Dir Psychol Sci. (2017) 26:10-5. doi: 10.1177/0963721416666518

24. Lydon-Staley DM, Bassett DS. The promise and challenges of intensive longitudinal designs for imbalance models of adolescent substance use. Front Psychol. (2018) 9:1576. doi: 10.3389/fpsyg.2018.01576

25. Valentine AZ, Hall SS, Young E, Brown BJ, Groom MJ, Hollis C, et al. Implementation of telehealth services to assess, monitor, and treat neurodevelopmental disorders: Systematic review. J Med Internet Res. (2021) 23:e22619. doi: 10.2196/22619

26. Curran PJ, Obeidat K, Losardo D. Twelve frequently asked questions about growth curve modeling. J Cogn Dev. (2010) 11:121-36. doi: 10.1080/15248371003699969

27. King KM, Littlefield AK, McCabe CJ, Mills KL, Flournoy J, Chassin L. Longitudinal modeling in developmental neuroimaging research: common challenges, and solutions from developmental psychology. Dev Cogn Neurosci. (2018) 33:54-72. doi: 10.1016/j.dcn.2017.11.009

28. te Brinke L, Menting A, Schuiringa H, Deković M, Weisz J, de Castro B. Emotion regulation training as a treatment element for externalizing problems in adolescence: a randomized controlled micro-trial. Behav Res Ther. (2021) 143:103889. doi: 10.1016/j.brat.2021.103889

29. Bennett TA, Szatmari P, Georgiades K, Hanna S, Janus M, Georgiades S, et al. Do reciprocal associations exist between social and language pathways in preschoolers with autism spectrum disorders? J Child Psychol Psychiatry. (2014) 56:874-83. doi: 10.1111/jcpp.12356

30. Georgiades S, Tait PA, McNicholas PD, Duku E, Zwaigenbaum L, Smith IM, et al. Trajectories of symptom severity in children with autism: Variability and turning points through the transition to school. J Autism Dev Disord. (2022) 52:392-401. doi: 10.1007/s10803-021-04949-2

31. Amaral DG, Vries PJ. COVID-19 and autism research: perspectives from around the globe. Autism Res. (2020) 13:844-69. doi: 10.1002/ aur.2329

32. Kasari C, Sturm A, Shih W. Smarter approach to personalizing intervention for children with autism spectrum disorder. J Speech Lang Hear Res. (2018) 61:2629-40. doi: 10.1044/2018_JSLHR-L-RSAUT-18-0029

33. Supekar K, Iyer T, Menon V. The influence of sex and age on prevalence rates of comorbid conditions in autism. Autism Res. (2017) 10:77889. doi: 10.1002/aur.1741

34. Sher KJ, Gotham HJ, Watson AL. Trajectories of dynamic predictors of disorder: Their meanings and implications. Dev Psychopathol. (2004) 16:82556. doi: $10.1017 /$ S0954579404040039

35. Granic I. Timing is everything: developmental psychopathology from a dynamic systems perspective. Dev Rev. (2005) 25:386407. doi: 10.1016/j.dr.2005.10.005

36. van Os J. The dynamics of subthreshold psychopathology: implications for diagnosis and treatment. Am J Psychiatry. (2013) 170:695-8. doi: 10.1176/appi.ajp.2013.13040474

37. Nelson B, McGorry PD, Wichers M, Wigman JT, Hartmann JA. Moving from static to dynamic models of the onset of mental disorder. JAMA Psychiatry. (2017) 74:528. doi: 10.1001/jamapsychiatry.2017.0001

38. Chen X, Gao W, Li J, You D, Yu Z, Zhang M, et al. A predictive paradigm for COVID-19 prognosis based on the longitudinal measure of biomarkers. Brief Bioinform. (2021) 22:bbab206. doi: 10.1093/bib/bbab206

39. Jenkins DA, Martin GP, Sperrin M, Riley RD, Debray TP, Collins GS, et al. Continual updating and monitoring of clinical prediction models: Time for dynamic prediction systems? Diagn Progn Res. (2021) 5:1-7. doi: 10.1186/s41512-020-00090-3

40. Davis SE, Greevy RA, Lasko TA, Walsh CG, Matheny ME. Detection of calibration drift in clinical prediction models to inform model updating. J Biomed Inform. (2020) 112:103611. doi: 10.1016/ j.jbi.2020.103611

41. Vivanti G, Prior M, Williams K, Dissanayake C. Predictors of outcomes in autism early intervention: why don't we know more? Front Pediatr. (2014) 2:58. doi: $10.3389 /$ fped.2014.00058 
42. Hofmann SG, Curtiss JE, Hayes SC. Beyond linear mediation: toward a dynamic network approach to study treatment processes. Clin Psychol Rev. (2020) 76:101824. doi: 10.1016/j.cpr.2020.101824

43. Pickles A, Le Couteur A, Leadbitter K, Salomone E, Cole-Fletcher R, Tobin H, et al. Parent-mediated social communication therapy for young children with autism (PACT): long-term follow-up of a randomised controlled trial. Lancet. (2016) 388:2501-9. doi: 10.1016/S0140-6736(16)31229-6

44. Geschwind DH, Sowinski J, Lord C, Iversen P, Shestack J, Jones P, et al. The autism genetic resource exchange: a resource for the study of autism and related neuropsychiatric conditions. Am J Hum Genet. (2001) 69:463. doi: 10.1086/321292

45. McPartland JC, Bernier RA, Jeste SS, Dawson G, Nelson CA, Chawarska K, et al. The autism biomarkers consortium for clinical trials (ABC-CT): scientific context, study design, and progress toward biomarker qualification. Front Integr Neurosci. (2020) 14:16. doi: 10.3389/fnint.2020.00016

46. Mund M, Johnson MD, Nestler S. Changes in size and interpretation of parameter estimates in within-person models in the presence of time-invariant and time-varying covariates. Front Psychol. (2021) 12:666928. doi: 10.3389/fpsyg.2021.666928

47. Curran PJ, Howard AL, Bainter SA, Lane ST, McGinley JS. The separation of between-person and within-person components of individual change over time: a latent curve model with structured residuals. J Consult Clin Psychol. (2014) 82:879-94. doi: 10.1037/a0035297

48. Tan X, Shiyko MP Li R, Li Y, Dierker L. A time-varying effect model for intensive longitudinal data. Psychol Methods. (2012) 17:6177. doi: 10.1037/a0025814

49. Asparouhov T, Hamaker EL, Muthén B. Dynamic structural equation models. Struct Eq Model. (2017) 25:359-88. doi: 10.1080/ 10705511.2017.1406803

50. Papageorgiou G, Mauff K, Tomer A, Rizopoulos D. An overview of joint modeling of time-to-event and longitudinal outcomes. Annu Rev Stat Appl. (2019) 6:223-40. doi: 10.1146/ annurev-statistics-030718-105048

51. Wright AG, Hallquist MN, Swartz HA, Frank E, Cyranowski JM. Treating cooccurring depression and anxiety: modeling the dynamics of psychopathology and psychotherapy using the time-varying effect model. J Consult Clin Psychol. (2014) 82:839-53. doi: 10.1037/a0034430

52. Lanza ST, Vasilenko SA, Russell MA. Time-varying effect modeling to address new questions in behavioral research: examples in marijuana use. Psychol Addict Behav. (2016) 30:939-54. doi: 10.1037/adb00 00208

53. Racine N, Plamondon A, Hentges R, Tough S, Madigan S. Dynamic and bidirectional associations between maternal stress, anxiety, and social support: the critical role of partner and family support. J Affect Disord. (2019) 252:1924. doi: 10.1016/j.jad.2019.03.083
54. Yuen HP, Mackinnon A, Nelson B. A new method for analysing transition to psychosis: Joint modelling of time-to-event outcome with time-dependent predictors. Int J Methods Psychiatr Res. (2017) 27:e1588. doi: $10.1002 / \mathrm{mpr} .1588$

55. Zhang Z, Reinikainen J, Adeleke K, Pieterse M, Groothuis-Oudshoorn C. Time-varying covariates and coefficients in Cox regression models. Ann Transl Med. (2018) 6:121. doi: 10.21037/atm.2018.02.12

56. Nestler S. An extension of the mixed-effects growth model that considers between-person differences in the within-subject variance and the autocorrelation. Stat Med. (2022) 41:471-82. doi: 10.1002/sim.9280

57. Cicchetti D, Toth SL. The past achievements and future promises of developmental psychopathology: the coming of age of a discipline. J Child Psychol Psychiatry. (2009) 50:16-25. doi: 10.1111/j.1469-7610.2008.01979.x

58. Loth E, Murphy DG, Spooren W. Defining precision medicine approaches to autism spectrum disorders: concepts and challenges. Front Psychiatry. (2016) 7:188. doi: 10.3389/fpsyt.2016.00188

59. Mandy W, Lai M-C. Annual research review: the role of the environment in the developmental psychopathology of autism spectrum condition. J Child Psychol Psychiatry. (2016) 57:271-92. doi: 10.1111/jcpp.12501

60. Ameis SH, Lai M-C, Mulsant BH, Szatmari P. Coping, fostering resilience, and driving care innovation for autistic people and their families during the COVID-19 pandemic and beyond. Mol Autism. (2020) 11:1-9. doi: 10.1186/s13229-020-00365-y

61. Lord C, Charman T, Havdahl A, Carbone P, Anagnostou E, Boyd B, et al The Lancet Commission on the future of care and clinical research in autism. Lancet. (2022) 399:271-334. doi: 10.1016/S0140-6736(21)01541-5

Conflict of Interest: The authors declare that the research was conducted in the absence of any commercial or financial relationships that could be construed as a potential conflict of interest.

Publisher's Note: All claims expressed in this article are solely those of the authors and do not necessarily represent those of their affiliated organizations, or those of the publisher, the editors and the reviewers. Any product that may be evaluated in this article, or claim that may be made by its manufacturer, is not guaranteed or endorsed by the publisher.

Copyright (c) 2022 Chen, Duku and Georgiades. This is an open-access article distributed under the terms of the Creative Commons Attribution License (CC BY). The use, distribution or reproduction in other forums is permitted, provided the original author(s) and the copyright owner(s) are credited and that the original publication in this journal is cited, in accordance with accepted academic practice. No use, distribution or reproduction is permitted which does not comply with these terms. 